\title{
Summarising big data: public GitHub dataset for software engineering challenges
}

\author{
Abdulkadir ŞEKER ${ }^{1, *(D)}$, Banu DİRI' (D) , Halil ARSLAN ${ }^{(\mathbb{D})}$, Mehmet Fatih AMASYALI $^{2}$ (D) \\ ${ }^{1}$ Sivas Cumhuriyet University, Department of Computer Engineering, Sivas/TURKEY \\ ${ }^{2}$ Ylldız Technical University, Department of Computer Engineering, İstanbul/ TURKEY
}

\begin{abstract}
In open-source software development environments; textual, numerical, and relationshipbased data generated are of interest to researchers. Various data sets are available for this data, which is frequently used in areas such as software engineering and natural language processing. However, since these data sets contain all the data in the environment, the problem arises in the terabytes of data processing. For this reason, almost all of the studies using GitHub data use filtered data according to certain criteria. In this context, using a different data set in each study makes a comparison of the accuracy of the studies quite difficult. In order to solve this problem, a common dataset was created and shared with the researchers, which would allow to work on many software engineering problems.
\end{abstract}

\section{Article info \\ History:}

Received:29.04.2020

Accepted:12.06.2020

Keywords:

GitHub,

Ghtorrent,

Big data,

MongoDB.

\section{Introduction}

One of the most common among cloud-based opensource versioning systems is GitHub. GitHub has become the world's largest code server that hosted more than 100 million developers and more than 40 million repos (https://en.wikipedia.org/wiki/GitHub). Repo is abbreviation for repository of projects, it can be thought of as a folder where we store projects. On GitHub-like platforms, development processes are distributed. Developers can make different contributions to projects from any location, via commenting, coding, opening issues, or fixing some bugs. By the results of these activities, large amounts of data are generated for researchers working on natural language processing and software engineering. In addition to these text data, from the social relationships of users with each other and with repos also extract different features. Thanks to these features, GitHub data is of great importance and interest for academic and commercial studies.

In studies with GitHub data, researchers obtain data via the GitHub API or use some datasets. The most widely used of these datasets is known as GHTorrent [1-3]. The GHTorrent dataset was developed in the software engineering department of the TU Delft University [4]. The dataset presents all the data on the platform as downloadable dumps. The sizes of the GitHub datasets reach very high levels as they contain information from the entire platform. Working with data in such sizes is a problem in itself. When studies using GitHub data are examined, it is seen that almost all of them have created specific sub-datasets. Researchers have used the data in their studies by filtering the data according to their problems and possibilities. It is not possible to compare the successes of even the studies on similar subjects since each researcher works on the dataset that he/she has created.

To address this problem in the literature, the copy of the GHTorrent dataset containing GitHub data up to 2015 was localized and filtered with certain parameters, duplicate data was extracted, new fields to link the data were added, and a MongoDB dataset was created. In this way, a data set, which is much smaller than the GitHub environment, has been produced and shared, which will offer the opportunity to work on the challenges of software engineering in many areas such as task, user, project, and software development. It is possible to use this dataset easily during the algorithm or model development phase. The dataset was shared as a MongoDB archive for the developed codes to be applied to big data without being changed.

\section{Definition of Problem}

In studies using GitHub data, filtered datasets are used. Even if the problems studied are the same, since the 
datasets are specific to each study, the accuracy of the claimed success rates has become controversial. For example, the works are given in Table 1 are all about pull request (PR). However, as can be seen, they have used different types of content and filtered data of different types. In order to provide a solution to this situation and to be able to conduct rapid experiments at the beginning of the studies, a small dataset was designed and produced to represent the big data.

Table 1. The Filtered Dataset from some studies

\begin{tabular}{|l|l|l|}
\hline Study & $\begin{array}{l}\text { Number } \\
\text { of repos }\end{array}$ & Type of filter \\
\hline Zhang-2014 [5] & 3587 & Has more than 100 PR \\
\hline Veen-2015 [6] & 475 & - \\
\hline Yu-2015 [7] & 40 & $\begin{array}{l}\text { Has more than 100 PR } \\
\text { and non-forked }\end{array}$ \\
\hline Junior-2018 [8] & 32 & $\begin{array}{l}\text { Has more than 100 PR } \\
\text { and at least 5 developers }\end{array}$ \\
\hline Zhao-2019 [9] & 74 & Develop with Java \\
\hline
\end{tabular}

GHTorrent consists of a lot of information about projects, definitions and contents of a problem (issue), comments, information about pull requests, code commit activities, repos that users follow, ... (Table 2).

Table 2. The Information of GHTorrent Dataset

\begin{tabular}{|l|l|}
\hline Domain & Collections \\
\hline User & $\begin{array}{l}\text { Users, Followers, Watchers, } \\
\text { RepoCollaborators }\end{array}$ \\
\hline Project & Projects, Forks \\
\hline Development & Commits, CommitComments \\
\hline Contribution & $\begin{array}{l}\text { Issues, IssueComments, } \\
\text { PullRequests, } \\
\text { PullRequestComments }\end{array}$ \\
\hline
\end{tabular}

This dataset offers GitHub data in different formats as SQL tables and MongoDB collections. In this study, a public subset of data was created with the version covering data up to 2015. With the MongoDB queries, some errors detected in the GHTorrent dataset have been corrected, free from repetitive data, link features that are noticed to be missing from the collections have been added, convert to a simple and easy-to-operate size and content. While creating the dataset, all the data (approximately 750 GB) stored as MongoDB collections were downloaded to the local environment and put through the processes mentioned above. These processes have been developed in MongoDB in order to suitable for big data. The queries used for all transactions are shared on GitHub;

https://github.com/kadirseker00/GitDataSCP.
Users is selected as the restrictive collection when filtering the dataset. A dataset has been created with all the information associated with the 100 selected users. All collections have been shared as CSV format, which is the most suitable in terms of size.

\section{Pre-Processes}

\subsection{Filtering}

The 100 most active users on GitHub from the Users collection were selected; According to list below url; https://gist.github.com/paulmillr/2657075

Based on these users, all data that has any relationship with them was filtered from other collections. The steps for filtering are shown in detail in Figure 1.

\subsection{Solving mistaken or missed data}

In order to obtain the dataset free from errors, it must be put through some process. In this section, the related MongoDB queries are given below each problem. Since the data selected in the dataset is planned in the user base, it is needed to determine the primary keys for collections. In this context, as a primary key (or distinctive feature); "user id" will be used on the data related to users, similarly "repo id" is used on the data associated with repos. Therefore, the existing problems with these fields must be eliminated.

Firstly, the documents that has null value of these key features have been removed (Figure 1).

db.Pull_requests.deleteMany(\{user_id: null $\})$

Figure 1 Remove null data

It has been noticed that the dataset contains duplicate documents that are thought created by users or retrieve mistakenly. Documents containing repeated data in key fields such as user id, repo id or full name were also removed.

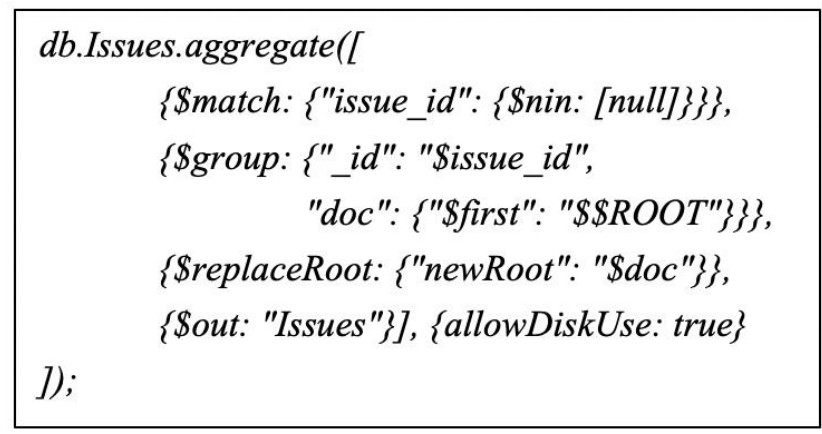

Figure 2 Remove duplicate data 
It has been noticed that the dataset contains duplicate documents that are thought created by users or retrieve mistakenly. Documents containing repeated data in key fields such as user id, repo id or full name were also removed (Figure 2).

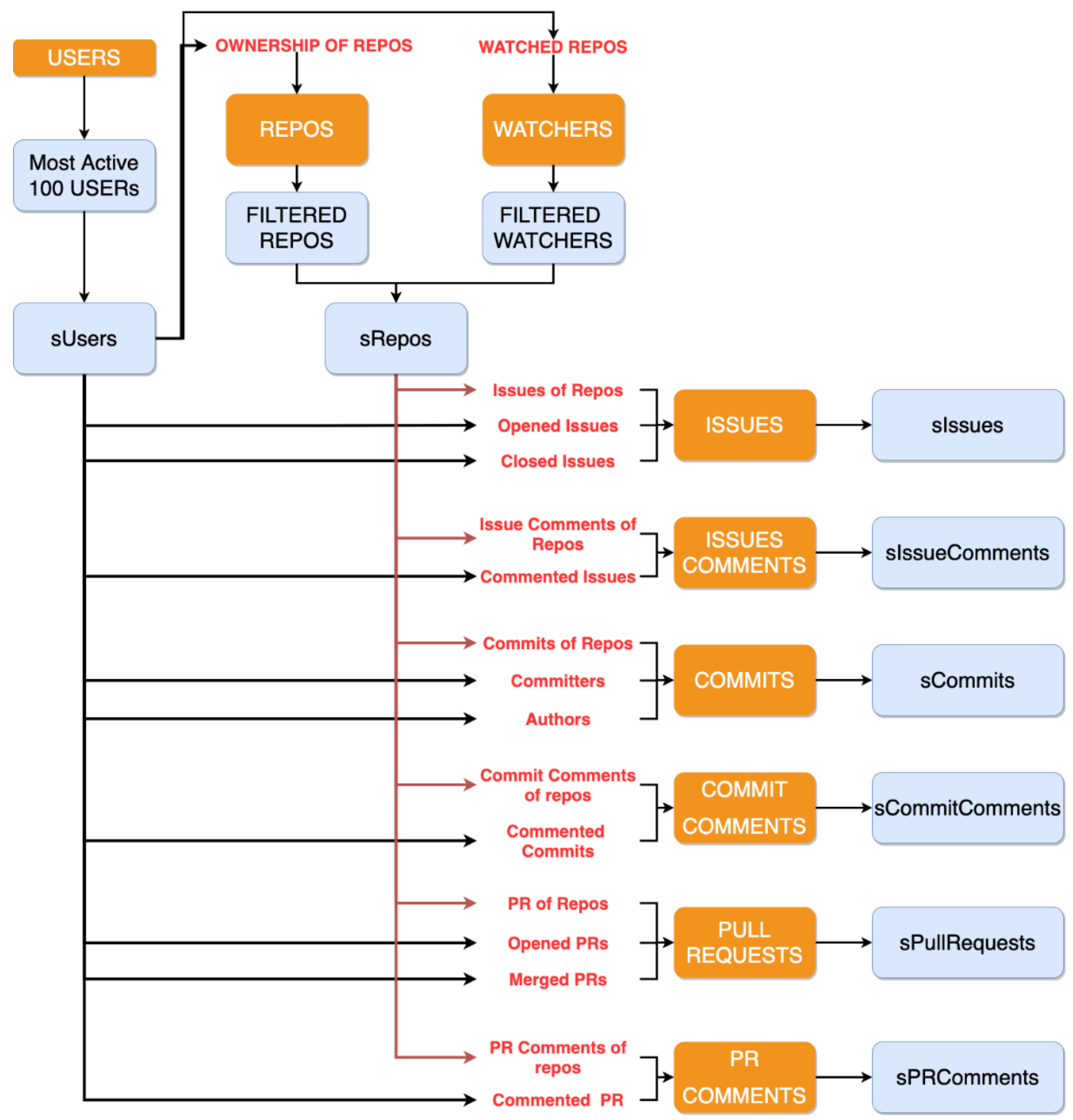

Figure 3 The filtering process with the most active 100 users

In some collections, it has been observed that the key fields do not exist or that their substitutes aren't sufficient.

a. Issues, Watchers collections have the owner and name fields of the repo. The full_ name field is created by combining two fields directly (Figure $4)$.

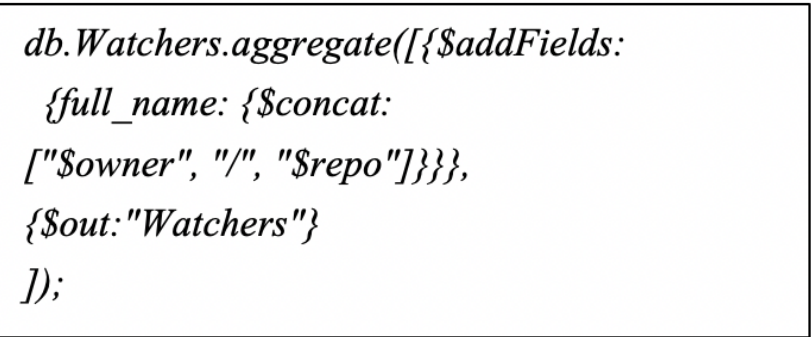

Figure 4 Create "full_name" field 
b. In the CommentCommits collection, there is no owner and name fields. To obtain them firstly has been parsed the repo url field and extracted these fields. Then full_name has been created as combining them (Figure 5). A sample repo url; https://github.com/johndue/projectX)

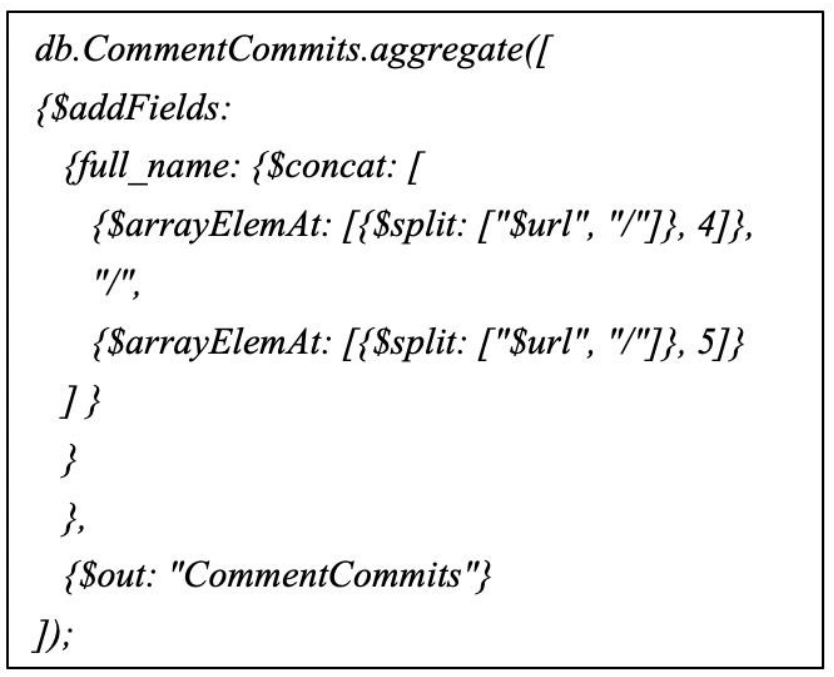

Figure 5 Extract "full_name" from url field c. After the process a or b, the field of repo_id has added to related collections as aggregate (join) with Repos collection on full_name. The query below means; find the documents from both collections which have the same full_name, then get the repo_id of this document from a collection and add it to a related document in the other collection (Figure 6).

d. Similarly, since there is no field of user_id in the Followers collection, this field was added by join with Users collection on login field. (login is the name of user in GitHub database.)

After handling these adding key or link fields process, the sub dataset has been created. The comparison of size between the GHTorrent and proposed filtered data set are given in Table 3. As can be seen from Table 3, working with huge data causes serious time losses, especially during the algorithm or model development phase. In this context, it is thought that the proposed filtered data set will provide researchers with a common data pool but will also save time in their studies. Dataset is published on page below;

https://github.com/kadirseker00/GitDataSCP

db.CommitComments.aggregate $([$

\{\$lookup:

\{from: "repos", let: \{item: "\$full_name"\}, pipeline: [

$\{$ \$match: $\{$ Sexpr: $\{$ \$eq: ["\$full_name","\$\$item"] $\}\}\}$, \{\$project: $\{$ "repo_id": 1$\}\}$

],

as: "fromComments"

\} ,

\{\$replaceRoot: \{newRoot:

$\{$ \$mergeObjects: [

\{\$arrayElemAt: ["\$fromComments",0]\},"\$\$ROOT"]\}

\}

\} ,

$\{$ project: $\{$ 'fromComments': 0\}\},

\{\$out: "FilteredCommitComments"\}

]);

Figure 6 Join with repos and other collection on "full_name" 
Table 3. The comparison of GHTorrent and proposed dataset

\begin{tabular}{|l|l|l|l|l|}
\hline & \multicolumn{2}{|c|}{$\sim$ Disk Size } & \multicolumn{2}{c|}{$\sim$ Number of documents } \\
\hline Name of Collection & GHTorrent & Proposed & GHTorrent & Proposed \\
\hline Users & $1 \mathrm{~GB}$ & $0.8 \mathrm{MB}$ & $5,000,000$ & 100 \\
\hline Repos & $30 \mathrm{~GB}$ & $45 \mathrm{MB}$ & $20,000,000$ & 40,000 \\
\hline Commits & $298 \mathrm{~GB}$ & $4 \mathrm{~GB}$ & $41,000,000$ & 500,000 \\
\hline Commit Comments & $1 \mathrm{~GB}$ & $90 \mathrm{MB}$ & $2,000,000$ & 250,000 \\
\hline Issues & $11 \mathrm{~GB}$ & $3 \mathrm{~GB}$ & $17,000,000$ & $3,000,000$ \\
\hline Issue Comments & $15 \mathrm{~GB}$ & $4 \mathrm{~GB}$ & $31,000,000$ & $9,000,000$ \\
\hline Pull Requests & $23 \mathrm{~GB}$ & $5 \mathrm{~GB}$ & $8,000,000$ & $1,500,000$ \\
\hline Pull Requests Comments & $6 \mathrm{~GB}$ & $1 \mathrm{~GB}$ & $5,000,000$ & $1,200,000$ \\
\hline Followers & $1 \mathrm{~GB}$ & $20 \mathrm{MB}$ & $7,000,000$ & 130,000 \\
\hline Forks & $8 \mathrm{~GB}$ & $5 \mathrm{MB}$ & $11,000,000$ & 7,000 \\
\hline Watchers & $7 \mathrm{~GB}$ & $8 \mathrm{MB}$ & $38,000,000$ & 50,000 \\
\hline RepoCollaborators & $1 \mathrm{~GB}$ & $2 \mathrm{MB}$ & $5,000,000$ & 4,000 \\
\hline
\end{tabular}

\section{Results and Discussion}

In this study, a common dataset was proposed to the researchers working for the solution of software engineering challenges as a result of filtering with the GHTorrent dataset that contains an up-to-date copy of GitHub data. All filtering operations were performed in accordance with the big data in MongoDB environment so that the current versions of the data set can be extracted, and extensions/enhancements can be made.

In the proposed dataset, the fields that will link between the collections have been added, the missing or repeated data noticed in the dataset have been removed.

As a result, a clear and easy-to-operate GitHub dataset has been generated. The GitHub link given in the study can be used for downloading the dataset. It is planned to studies on the software engineering challenges with this proposed dataset.

\section{References}

[1] V. Cosentino, J. Luis, and J. Cabot. Findings from GitHub: methods, datasets and limitations. Proceedings of the 13th Int. Workshop on Mining Softw. Repositories, (2016), 137-141.

[2] V. Cosentino, J. L. Canovas Izquierdo, and J. Cabot. A Systematic Mapping Study of Software Development With GitHub, IEEE Access, 5 (2017) 7173-7192.
[3] Z. Kotti and D. Spinellis. Standing on shoulders or feet?: the usage of the MSR data papers, Proceedings of the 16th Int. Conference on Mining Software Repositories, (2019) 565-576.

[4] G. Gousios. The GHTorrent dataset and tool suite, Proceedings of the 10th Working Conf. on Mining Soft. Repositories, (2013) 233-236.

[5] Y. Zhang, G. Yin, Y. Yu, and H. Wang. Investigating social media in GitHub's pullrequests: a case study on Ruby on Rails, Proceedings of the 1st International Workshop on Crowd-based Soft. Development Methods and Technologies - CrowdSoft 2014 (2014) 37-41.

[6] E. van der Veen, G. Gousios, and A. Zaidman. Automatically Prioritizing Pull Requests, 2015 IEEE/ACM 12th Working Conference on Mining Soft. Repositories. (2015) 357-361.

[7] Y. Yu, H. Wang, V. Filkov, P. Devanbu, and B. Vasilescu. Wait for It: Determinants of Pull Request Evaluation Latency on GitHub. 2015 IEEE/ACM 12th Working Conference on Mining Software Repositories, (2015) 367-371.

[8] M. L. de L. Júnior, D. M. Soares, A. Plastino, and L. Murta. Automatic assignment of integrators to pull requests: The importance of selecting appropriate attributes, J. Syst. Softw., 144 (2018) 181-196.

[9] G. Zhao, D. A. da Costa, and Y. Zou. Improving the Pull Requests Review Process Using Learning-to-rank Algorithms, Empir. Softw. Eng., (2019) 1-31. 